\title{
Why BICA is Necessary for AGI
}

\author{
Alexei V. Samsonovich \\ Krasnow Institute for Advanced Study, George Mason University \\ 4400 University Drive MS 2A1, Fairfax, VA 22030-4444, USA \\ asamsono@gmu.edu
}

\begin{abstract}
The challenge of creating AGI is better understood in the context of recent studies of biologically inspired cognitive architectures (BICA). While the solution is still far away, promising ideas can be derived from biological inspirations. The notions of a chain reaction, its critical mass and scaling laws prove to be helpful in understanding the conditions for a self-sustained bootstrapped cognitive growth of artifacts. Empirical identification of the critical mass of intelligence is possible using the BICA framework and scalability criteria.
\end{abstract}

Keywords: cognitive architectures, self-regulated learning, human-level intelligence, scaffolding, critical mass

\section{Why BICA Challenge Is a Necessary Step}

The challenge of artificial intelligence formulated more than half a century ago (McCarthy et al. 1955/2006) turned far more complicated and more vital for us than originally thought. Today we understand that a similar situation exists with the BICA challenge. This challenge is to create a biologically inspired cognitive architecture (BICA) capable of mimicking the human cognition and learning. It can be viewed as a more focused and constrained version of the general artificial intelligence (AGI) challenge. At the same time, the BICA challenge is primarily targeting the core of higher cognition. Could the BICA challenge be a necessary step in solving the AGI challenge? Biological solutions are not always suitable for engineering tasks: e.g., airplanes do not flap wings and would not benefit from doing this. Biological inspirations, however, appear to be necessary for reaching AGI, because in this case biology provides the only known model of a solution.

The original, informally stated goal of the DARPA IPTO BICA program (2005-2006) was to capture the 'magic' of human cognition, while no clear understanding of what this 'magic' is was available in 2005. Initially, the focus was on integration, biological fidelity, and testdriven design, with a special attention paid to humanspecific higher cognitive abilities: meta-cognition, selfawareness, episodic memory, emotional intelligence, theory of mind, natural language and social capabilities. By the end of the funded period it became clear that the human-like learning is the key for solving the challenge,

Copyright (C) 2008, The Second Conference on Artificial General Intelligence (agi-09.org). All rights reserved. and 'capturing the magic' amounts to reproducing the phenomenon of human cognitive growth from a two-year old to an adult, using various forms of scaffolding and guidance by human instructors. This scenario became a prototype for the big BICA Challenge intended for Phase II, which, unfortunately, was canceled.

Recently, BICA alumni and other interested researchers gathered in Arlington in a AAAI 2008 Fall Symposium on BICA (http://binf.gmu.edu/ asamsono/bica/), trying to understand what went right and what went wrong with the BICA program, and what can we learn from this enterprise. During the symposium, we learned that we are still interested in the BICA Challenge and are dedicated to solving it, because we view it as a critical stepping stone on the road to AGI (Samsonovich \& Mueller, 2008).

The BICA Challenge understood as explained above has a potential impact of its solution extending far beyond immediate military or industrial goals. Its objective is to bridge the gap separating artificial and natural intelligence: the gap in autonomous cognitive growth abilities. This main dimension of the gap underlies its other dimensions, including robustness, flexibility and adaptability. In this sense, solving the BICA Challenge is a necessary, critical step in reaching AGI. It is argued below that using a BICA as a basis of a solution is also necessary.

\section{Critical Mass Hypothesis and Biology}

The critical mass hypothesis can be formulated as follows. By enabling the core human-like-learning mechanisms in artifacts, one can initiate a "chain reaction" of development of knowledge, skills and learning capabilities in artifacts. Agents learn how to learn and how to improve themselves. Given the right embodiment, embedding and scaffolding, this hypothetical process of bootstrapped cognitive growth will continue to a point when artifacts reach a human level of AGI. If this concept makes sense, then it is natural to accept that the cognitive chain reaction is characterized by a critical mass: a minimal set of architectural components and mechanisms, cognitive functions, features of the embodiment, interface, environment and scaffolding that together make the reaction possible, self-sustainable and sufficiently scalable. If this is the case, then identifying the critical mass (or, to be more parsimonious, any feasible supercritical mass) would constitute a substantial part of a solution to the BICA/AGI challenge. 


\section{Why and How Biology Gets Involved}

Despite the impressive recent progress in many fields of artificial intelligence, as of now there is no clear prospect of a self-sustainable cognitive chain reaction with its critical mass, scaling laws and other parameters derived from studies of artificial intelligence. There is only one known example of a cognitive chain reaction: natural human development. As a matter of biological fact, we know that a normal human child under typical social conditions possesses a supercritical mass, while a lower animal or an out-of-shelf computer do not; therefore, there must be a critical mass somewhere in between. In order to identify it, one can start moving down from the known positive example, taking out functional components one by one. To do this, one may study human pathologies that prevent normal cognitive development. While a rigorous study of this sort would be difficult, it should be pointed here that children deprived of certain seemingly vital sensory and/or action abilities can nevertheless grow cognitively to an adult level. On the other hand, the ability to acquire some form of language interactively and the ability to exercise voluntary behavior appear to be critical for cognitive growth (e.g., Thelin \& Fussner 2005).

Even if it is possible to reduce the AGI (or BICA) challenge to a language acquisition challenge for an embodied agent, this step does not make the task substantially easier. E.g., there is currently no good idea of how to approach passing the Turing test, or what tests would be useful to pass in order to ensure the language acquisition capacity. There is no clear understanding of how to build scaffolding for the growing agent, or what should be the driving force in its cognitive growth. Here biology provides another hint. The development and survivability of a biological organism depends on a number of "built-in" drives: from lower drives, such as hunger, pain and pleasure, to higher drives, such as curiosity and appreciation of beauty. It is reasonable to think that a core set of drives should be a part of the critical mass.

\section{Defining and Detecting the Critical Mass}

There are many questions related to identification of the critical mass in terms of BICA; here are some of them. What should we borrow from studies of the acquisition of language by young children? How the agent should develop an understanding of concepts associated with linguistic constructs? Is language processing an underlying capacity for other faculties like symbolic processing, or vice versa? What kinds of architectural mechanisms should be involved in symbolic and subsymbolic processing? What are the general critical mass requirements for memory systems, kinds of representations and principles of information processing? What cognitive abilities should be innate (preprogrammed), and which of them should develop through learning? What can we say regarding the ladder, i.e., the curriculum for artifacts understood as the sequence of tasks, paradigms and intermediate learning goals that will scaffold their rapid cognitive growth up to the human level? An answer to these questions will be provided by the definition of a critical mass.

Assuming that there is a threshold level of intelligence that enables bootstrapping, we should expect certain scalability of the phenomenon. The notion of a chain reaction predicts an exponential scaling law. Therefore, scaling laws and metrics associated with them can be taken as a basis for the main criterion for a critical mass. It is therefore interesting and necessary to conduct a set of experiments with cognitive growth demonstrations using the BICA framework, starting from simplistic "toy" examples and gradually relaxing their limitations. Finally, the answer should be given in the form of a BICA, because the only known prototype exists in biology.

\section{A BICA-Based Roadmap to AGI}

A specific approach to solving the BICA Challenge (Samsonovich et al. 2008) was developed by our George Mason University team based on a combination of the GMU BICA cognitive architecture and the concept of selfregulated learning, or SRL (Zimmerman 2002). The key idea is to use GMU BICA as a model of SRL that will be dynamically mapped to the student mind. This approach will allow us to develop a faithful model of student SRL, to be used in education and in artificial intelligence, starting with intelligent tutoring systems that will be used as SRL assistants. While this is the current agenda, our eventual goal is to build Robby the Robot that can learn virtually everything like a human child.

\section{References}

McCarthy, J., Minsky, M., Rochester, N., and Shannon, C. (1955/2006). A Proposal for the Dartmouth Summer Research Project on Artificial Intelligence. AI Magazine, 27 (4) 12-14.

Samsonovich, A. V., Kitsantas, A., and Dabbag, N. (2008). Cognitive Constructor: A Biologically-Inspired SelfRegulated Learning Partner. In Samsonovich, A. V. (Ed.). Biologically Inspired Cognitive Architectures. Papers from the AAAI Fall Symposium. AAAI Technical Report FS-0804, pp. 91-96. Menlo Park, CA: AAAI Press.

Samsonovich, A. V., and Mueller, S. T. (2008). Toward a growing computational replica of the human mind. In Samsonovich, A. V. (Ed.). Biologically Inspired Cognitive Architectures. Papers from the AAAI Fall Symposium. AAAI Technical Report FS-08-04, pp. 1-3. Menlo Park, CA: AAAI Press.

Thelin, J. W. and Fussner, J. C. (2005). Factors related to the development of communication in CHARGE syndrome. American Journal of Medical Genetics Part A, 133A (3): 282-290.

Zimmerman, B. J. (2002). Becoming a self-regulated learner: An overview. Theory into Practice, 41 (2): 64-70. 